\title{
Acceptability of a physiotherapeutic pain school treatment in trauma-affected popu- lations in the Middle Eastern \& Northern African region
}

\author{
Anne-Mette Karrer ${ }^{1}$, Ane Kirstine Viller Hansen², Marie Louise D. Oestergaard ${ }^{3}$ \\ and Linda Nordin 4
}

\section{Key points of interest}

- A mixed method study of a physiotherapeutic pain treatment for persistent pain conditions (Pain School), showed acceptance and feasibility among physiotherapists in the Middle Eastern and Northern African (MENA) region.

- Initial quantitative outcome of this treatment, in the MENA region, also indicated positive results in traumatised individuals.

- Limitations were found in possible author biases and in not having a control group.
1) MSc, DIGNITY: Danish Institute Against Torture, Copenhagen, Denmark.

Correspondence to: amk@dignity.dk

2) MSc. DIGNITY: Danish Institute Against Torture, Copenhagen, Denmark

3) MSc. DIGNITY: Danish Institute Against Torture, Copenhagen, Denmark

4) PhD, DIGNITY: Danish Institute Against Torture, Copenhagen, Denmark; and Department of Psychology, Lund University, Lund, Sweden

\begin{abstract}
Introduction: The aim of this study was to evaluate the acceptability of a physiotherapeutic pain treatment (Pain School), focusing on patient education, physical exercises and self-reliance, and a capacity building program developed for MENA based physiotherapists working with pain and trauma-affected populations.

Method: Qualitative interviews with seven physiotherapists participating in the Pain School training program. Preliminary results of the Pain School treatment were also analyzed in 38 patients suffering from persistent pain and trauma-related stress.

Results: The qualitative analysis showed good feasibility and high acceptance among the participating physiotherapists. The pre- to post-treatment evaluation of Pain School, also gave an indication of positive treatment effects.

Limitations: Lack of mental health measures, author biases (authors carrying out training, interviews and analyzing qualitative results) and no control group.
\end{abstract}

Keywords: persistent pain, traumatic stress, feasibility study, physiotherapy, MENA.

\section{Introduction}

Being a refugee, having survived traumatic experiences and living under continuous stressful conditions, such as poverty, loss of status, and limited access to health services 
and education, can lead to long-lasting negative implications on a person's health (Pacella et al., 2013; Rometsch-Ogioun El Sount et al., 2019), the most common being posttraumatic stress disorder (PTSD), anxiety, depression, poor sleep and persistent pain (Husak \& Bair, 2020; Nicol et al., 2016; Williams \& Alayarian, 2019).

The lack of pain specific treatment methods in combination with large numbers of refugees in regions such as the Middle East and North Africa (MENA), indicate a crucial need to develop cost-effective, evidence-based methods that address persistent pain, as well as educating health professionals in handling these complex cases.

The aim of this mixed method study is to: (1) Evaluate the acceptability and feasibility of Pain School, a physiotherapeutic pain treatment, developed and contextualized during a capacity building program for MENA-based physiotherapists; (2) Evaluate the clinical trainings of physiotherapists delivering the treatment; (3) In a preliminary fashion, investigate the pilot results of the Pain School treatment in patients suffering from persistent pain and trauma-related stress.

\section{Study specific theoretical background}

In 2019, the International Association for the Study of Pain (IASP) introduced The International Classification of Diseases' (ICD-11) definition of chronic primary pain. Chronic primary pain is a persistent pain condition, where the pain is, not better accounted for by any other specific classified disease (Perrot et.al., 2019). Recent years of pain and neuroplasticity research supports a change in terminology from chronic pain to persistent pain by suggesting that persistent pain conditions are indeed not necessarily chronic. As persistent pain can decrease and/or stabilize over time (Abbey, 2015; Moseley \& Butler, 2015;
Moseley \& Butler, 2017). A shift from a biomedical to a biopsychosocial understanding of pain, in recent years, is clearly seen in IASP's definition of pain as "An unpleasant sensory and emotional experience associated with, or resembling that associated with, actual or potential tissue damage" (IASP, 2019).

When both PTSD and pain are present, the severity, duration, and functional impact tend to increase (Harlacher et al., 2016; Ruiz-Parraga \& Lopez-Martinez, 2014). It has been suggested that not only PTSD, but also depression and anxiety may interact with pain through a combination of mechanisms (Brennstuhl et al., 2014; McAndrew et al., 2019). An attempt to illustrate the complexity is Amundson \& Katz's shared vulnerability model (Figure 1).

The model represents a biopsychosocial understanding of pain and suggests that the traumatic events, such as violence, torture or displacement, can create an emotional response, leading to a modulation in the nervous that affects the response system and overall pain perception. The alertness in the nervous system that is present in both trauma-related responses and pain conditions creates increased sensitivity and can produce dysfunctional neuroplastic changes. What seems to be an over-evaluation of risk of harm, creates a hypersensitive nervous system leading to a low threshold for daily stressors and bodily sensation, with increased pain and stress reactions, as a result (Asmundson \& Katz, 2009; Defrin et.al., 2017; Nordin \& Perrin, 2019). 
Figure 1. Shared vulnerability model.

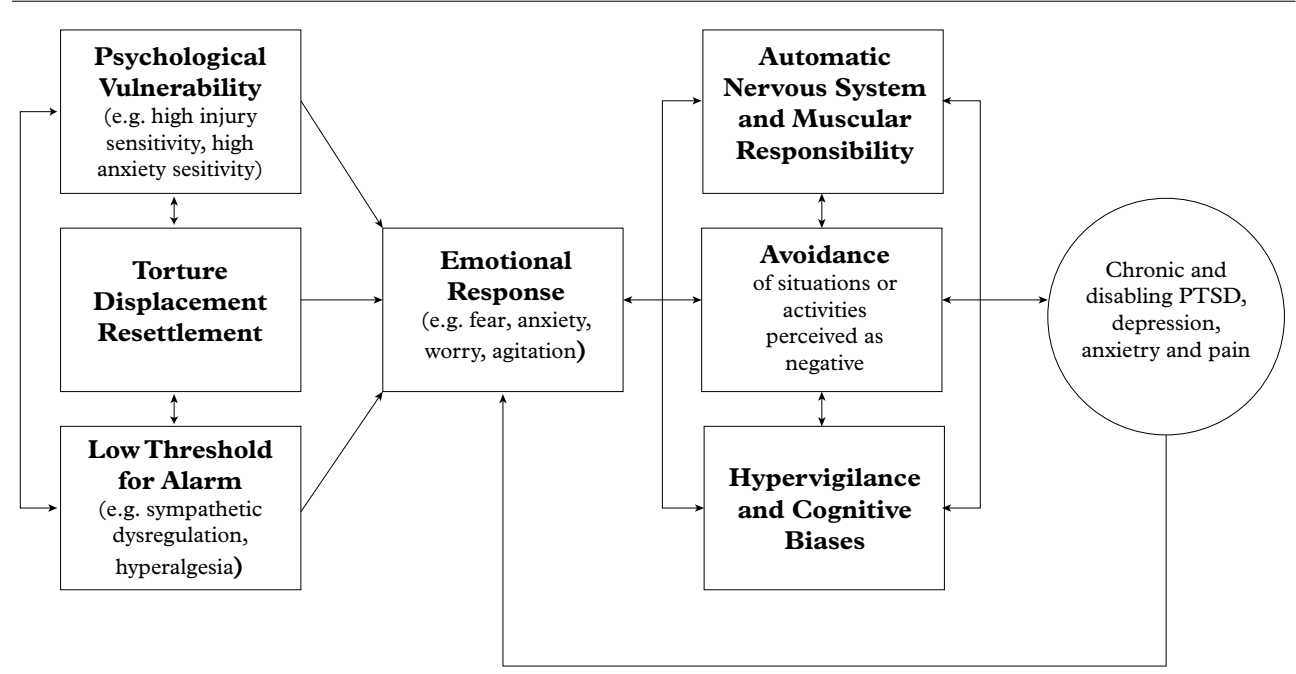

Figure 1. Shared vulnerability model. From Asmundson GJG, Abrams MP, Collimore KC: Pain and anxiety disorders, in Health behaviors and physical illness in anxiety and its disorders: Contemporary theory and research. Edited by Zvolensky MJ, Smits JAJ. New York: Springer, 2008, pp 207-235, p. 216. Copyright 2008.

\section{Symptomology and treatments}

Persistent pain conditions among refugee populations, living in asylum in the western world, is known as a significant contributor to decreased quality of life, functioning and disability (Buhman et al., 2014, Nordin, 2020). Persistent pain and trauma-related stress enhances "fear-avoidance" behavior (Nordin \& Perrin, 2019; Vlaeyen \& Linton, 2000), leading to general inactivity, which again negatively affects mental and physical health (Boakye et al., 2016); Martinez-Calderon et al., 2020). The documentation of persistent pain and somatic distress, along the expected co-occurrence with mental health conditions and malfunctioning among refugees in the Middle East, or bordering countries, is limited. A recent study, assessing Syrian refugees in Turkey, (McGrath et al., 2020) indicates the same symptomology and a 2008 study concluded that such co-occurrence seems to be similar across cultures and regions of the world (Tsang et al., 2008).

A Cochrane Review from 2017 (Baird et al., 2017) revealed only three documented attempts to address persistent pain in torture survivors. The treatments that were evaluated were cognitive behavioral therapy (CBT) with biofeedback, in combination with either physiotherapy group treatment or physiotherapy home exercises, versus waiting list (Liedl et al., 2011; Wang et al., 2017) and complex manual therapy versus self-treatment (Kim \& Yu, 2015). None of the studies demonstrated a reduction in pain, and only the manual therapy study claimed, at the end of treatment, to have reduced disability and distress. The review also concluded that the prevalent persistent pain condition tends to be overlooked by mental health professionals and therefore few attempts have been made to explore effective treatments for the traumatized refu- 
gees, including survivors of torture, especially in regions outside the western world.

The shift from a biomedical understanding to a biopsychosocial understanding of pain has changed the clinical approaches in pain rehabilitation. IASP recommends that the treatment of non-malign, persistent pain conditions in torture survivors, should contain components of pain education, physical therapy, self-management and behavioral aspects of adaptation to pain (Amris \& Jansen, 2019), which equals IASP general guidelines for all pain rehabilitation. Understanding the biopsychosocial reasons for pain, as well as slowly re-learning that it is safe to stay active, seem to stabilize the overall conditions (Louw et al., 2016). Studies conducted in the western world have shown that protocols containing pain education and exercise therapy for patients suffering from persistent pain have a positive impact on self- perceived health and disability, pain intensity, physical function, life quality and avoidance behavior amongst others. However, lack of replicable protocols and consistency in outcome measures makes the studies hard to compare (Chipchase et al., 2012; Malfliet et al., 2018; Pardo et al., 2018).

Three clinical approaches often used within pain and mental health rehabilitation are: Basic Body Awareness Therapy (BBAT); Acceptance, Commitment Therapy, ACT and Pacing principles. BBAT is a Scandinavian developed body-mind physiotherapy method (Gyllensten et al., 2018). Studies find that BBAT has a positive effect on pain, stress, depression and physical function (Blaauwendraat et al., 2017; Bravo et al., 2018.; Stade et al., 2015). Pacing principles (Moseley \& Butler, 2013, p.118-125) provide a guideline on how to economize strength and endurance by working and resting intermittently during daily activities and physical training, with the aim to stabilize and avoid pain, stress and fatigue outbursts. Both Pacing principles and BBAT exercises aim to decrease the need to use a fear-avoidance strategy and thereby enable a person to stay active in their daily life. ACT is an acceptance-based, action-oriented approach that originates from the third wave of cognitive behavioral therapies (CBT) (Dahl \& Lundgren, 2006; Dahl et al., 2004). ACT has proven to have effect on pain, depression and anxiety (A-Tjak et al., 2015; Zhenggang et al., 2020). ACT focuses on the overall "here and now" condition and not the cause of the pain, anxiety or depression. ACT promotes the individual to plan, for them, meaningful and necessary activities despite their conditions. ACT, as BBAT, uses mindfulness and body awareness to facilitate, acceptance, resilience and self-reliance.

\section{Method}

\section{Ethical Considerations}

The study follows the World Medical Association's (WMA) Declaration of Helsinki 1964, as updated most recently in 2013: International Ethical Guidelines for Biomedical Research Involving Human Subjects, including research on identifiable human material and data (WMA, 2013). DIGNITY - Danish Institute against Torture's internal ethical committee provided approval for the treatment manual and the monitoring and evaluation (M\&E) tool. At this stage of the feasibility study no further approval was applied for, since the content of the treatment has been part of clinical practices for the target group in Denmark for years, with no known negative outcomes. Informed consent forms were signed by both physiotherapists receiving training and patients receiving treatment.

\section{Study Design}

The study follows the new Medical Research 
Council's (MIC) guidelines for developing and testing complex interventions (Craig et al., 2008). The guideline contains IV phases; I: Development II: Piloting/Feasibility III: Evaluation and IV Implementation. This study represents a phase I and II trial of a complex intervention, applying a mixed method design.

Phase I: Development and configuration of the pain school treatment

DIGNITY initiated a capacity building program for physiotherapists working in nongovernmental organizations (NGOs) in the MENA region. The purpose of the program was to provide training for physiotherapists who are treating trauma-affected patients that also suffer from persistent pain. The NGOs expressed an urgent need for new, cost-effective, and replicable ways to treat the large numbers of refugees and/or torture survivors, who continued to seek treatment for severe pain.

In collaboration, DIGNITY and the NGOs identified areas in which the physiotherapists needed to increase their competencies. It was observed that among the physiotherapists there was an overall biomedical understanding of pain conditions. Many were using manual and electrical techniques (i.e. TENS, ultra-sound) to treat pain while few used exercise therapy and patient education. Additionally, many also concentrated their treatment on relieving symptoms, and did not include concepts of empowerment, prevention or self-reliance as part of their treatment.

An already existing, not published, Danish Pain School treatment guide was manualized and adapted throughout the first half of a training program, using feedback from the physiotherapists in training to contextualize and include enough materials to support their clinical praxis when providing the Pain
School treatment and to make the treatment replicable.

Population: The first group of trainees consisted of 12 physiotherapists (seven females, five males) of which 10 physiotherapists completed the program. The trainings, $4 \times 5$ days, took place in Amman, Jordan. The training was carried out in English, with the support from Arab and French interpreters. The physiotherapists worked in health clinics and in refugee camps, based in Tunisia, Morocco, Lebanon and Jordan. They had between one and 30 years of clinical experience, with the majority having 5-8 years of experience.

The training program was designed to give the physiotherapists clinical competences to apply the Pain School treatment for groups and individuals. The trainings consisted of lectures, group work, roleplay, physical exercise training and supervision. The program contained one written and one practical evaluation and a required a number of case-presentations during online supervisions between trainings. The evaluations were only used to ensure relevant support for the individual physiotherapist in areas they found difficult and to motivate for self-study.

Configuration of manual: The Pain School treatment manual aims to empower trauma and pain-affected patients to live meaningful and engaged lives, despite pain and trauma-related stress conditions. The treatment consists of 10 manualized sessions, containing three main components: 1) Patient education with the aim to increase the patient's general understanding of physical and mental challenges and body-mind interaction, 2) Physiotherapeutic exercises to decrease fear-avoidance and increase basic body function and awareness, 3) Active planning for behavioral changes to decrease fear-avoidance strategies and enhance daily functioning, selfcare and self-reliance. The duration of each group session is $2 \times 45-$ 
minutes and 60 minutes for individual sessions. Each session is planned to contain an equal amount of time for all three components. The educational topics addressed in the manual are 1) Pain Mechanisms, 2) Pain and Stress, 3) Pain and Sleep, 4) Pain, activity and daily living (Table 1 ).

The patient education is presented, in a lay-person friendly manner with the aim to provide an understanding of pain mechanisms, stress and co-related challenges such as poor sleep and inactivity (Moseley \& Butler, 2017). The physiotherapeutic exercises are inspired by BBAT (Gyllensten et al., 2018) and Pacing principles (Moseley \& Butler 2013, p. 118-125). Through the calm and rhythmical movements, the exercises provide practice on how to economize strength and endurance by working and resting intermittently during daily activities and physical training, with the aim to stabilize and avoid pain, stress and fatigue outbursts. Principles from ACT are used to promote active planning for behavioral changes. In each session, the patients make plans for home practice and exercises between the sessions to integrate what they learn in their daily life. Metaphors and analogs are used to promote understanding of the topics addressed and self-reflection.

\section{Phase 2: Feasibility Pilot-Study}

The Pain School treatment was evaluated with a mixed-method analysis using quantitative analysis of the initial treatment effect and qualitative interviews with physiotherapists (receiving training and delivering the treatment). All participants complete standardized measures of mental and physical health

Table 1: Session content Pain School treatment manual

\begin{tabular}{|c|c|}
\hline Session 1: INTRODUCTION & $\begin{array}{l}\text { - Why are we here and what will we learn and } \\
\text { explore? } \\
\text { - Introduction to the treatment and structure of the } \\
\text { sessions } \\
\text { - Tryout the exercises and plan first home-exercise }\end{array}$ \\
\hline Session 2 and 3: PAIN MECHANISMS & $\begin{array}{l}\text { - What is acute pain? } \\
\text { - What is persistent pain? } \\
\text { - How can I understand, and should I react to acute } \\
\text { and persistent pain? }\end{array}$ \\
\hline Session 4 and 5: PAIN AND STRESS & $\begin{array}{l}\text { - What is trauma-affected stress? } \\
\text { - How does stress and anxiety affect how and when } \\
\text { we feel pain? } \\
\text { - What is catastrophizing? }\end{array}$ \\
\hline Session 6 and 7: SLEEP AND PAIN & $\begin{array}{l}\text { - Why do we need our sleep? } \\
\text { - What can I do to improve my sleep? } \\
\text { - What can I do to reduce pain and stress during } \\
\text { sleep? }\end{array}$ \\
\hline $\begin{array}{l}\text { Session } 8 \text { and 9: ACTIVITY, DAILY } \\
\text { LIVING AND PAIN }\end{array}$ & $\begin{array}{l}\text { - How do I live with pain? } \\
\text { - How do I balance my level of activity? }\end{array}$ \\
\hline
\end{tabular}


(described below) pre- and post-treatment. Monitoring and evaluation tool for Pain School treatment: Pain severity and pain interference were assessed using the nine item, short-form version of the Brief Pain Inventory (BPI) (Cleeland \& Ryan, 1994). Item 1 asks whether the person has experienced any pain over the past week. They are then presented with a two-dimensional representation of the human body (front and rear projections) and asked to shade in areas where they experience pain, making it possible to assess the total number of shaded areas. The next four items assess the worst, least, average, and current pain interference $(0=$ No Pain, $10=$ Worst Pain Imaginable). Two items assess medication use and the degree of relief from pain when using the medication $(0 \%=$ No relief, $100 \%$ $=$ Complete relief). Item 9 asks the respondent to rate interference from pain $(0=$ No interference, $10=$ Complete interference) in seven different areas of life (general activity, mood, mobility, work, relations with others, sleep, and enjoyment of life). Pain severity (four items) and pain interference (seven items) scores are the mean of the $0-10$ ratings for their respec- tive items. The BPI has been found to possess high levels of internal consistency for both pain severity and pain interference (Cronbach $\alpha$ $=.85$ and .88), and to be valid for use in medical and psychiatric populations across cultures (Cleeland \& Ryan, 1994; Tan et al., 2004; Turk et al., 2003). The internal reliability coefficients for the pain severity and pain interference scales in the current sample were Cronbach $\alpha=.91$ and .93 .

Body awareness was assessed using the nine question Body Awareness Scale-Experience, (BAS-E) questionnaire_(Gyllensten, \& Mattsson, 2011; Hedlund et al., 2016). BAS-E gives information about the patient's own views on how the body is functioning in their daily life. Items focusing on 1) the experience of the body, 2) muscle tension, 3) pain and discomfort, 4) ability to perform daily activities, 5) exercise habits, 6) relationship to appearance, and 7) breathing, as well as 8) subjective belief in if the overall conditions can improve and 9) coping strategies in daily life. The scale ranges from $0-3$, where 0 equals no problems and 3 equals extreme problems. Each question offers narrative answers on each

Table 2: Session-outline for session 2-10 (60 minutes or 2 x 45 minutes)

Starting the session (5-10 minutes)

Breathing and grounding exercise

Follow-up from last session (5-15 minutes)

Questions to be explored (See table 1)

Education and sharing on today's topic (10-15 minutes)

BBAT inspired exercises (minimum 20 minutes)

Education and sharing on today's topic (10-15 minutes)

Finishing the session (10/15 minutes)

Short plan for home practice (ACT and pacing principles are used)

Progressive muscle relaxation

If the session is carried out in $2 \times 45$ minutes a break is placed where it seems most relevant. 
level (0-3), of which the patient chooses the answer that equals their subjective experience the best. BAS-E was developed as a part of the Body Awareness Scale Movement Quality and Experience (BAS MQ-E), that also contains a movement quality assessment and a qualitative interview about experience during movement. The movement quality assessment is not part of the Pain School assessment tool and therefore not included in this study. The BAS-E questionnaire has, as part of the BAS MQ-E, been validated for patients with musculoskeletal pain, schizophrenia, affective disorders, as well as for healthy adults. The interrater reliability and concurrent validity were found acceptable (Sundén et al., 2016). BAS MQ-E has also been found applicable and useful as a measure of bodily symptoms in patients with PTSD (Nyboe et al., 2016).

The English and Arabic translations of the BAS-E questionnaire were not validated. Translations were made by experienced translators in close collaboration with BBAT specialists in Denmark and Sweden and the first author. The questionnaire is widely used in rehabilitation centers treating Middle Eastern patients in Scandinavia but has not been culturally validated yet. Self-rated health was examined with the single-item question: "In general, would you say that your health is: excellent, very good, good, fair, or poor ?", on a five-point scale where 1 equals excellent and 5 equals poor. This question was included, because it has been established as a strong predictor of mortality and morbidity (Idler \& Benyamini, 1997; Schnittker \& Bacak, 2014).

Exposure to trauma and torture was examined with two questions; "Have you ever experienced traumatic war events?" and "Have you ever experienced torture?". The physiotherapists were instructed to obtain this information from the referring doctor, psychologist or medical journal and if this information was not available, to leave the question un-answered. If the physiotherapists were already trained to address these kinds of sensitive questions, they could also address it directly with the patient.

Statistical analyses: All analyses were conducted with a two-sided level of significance $(p<0.05)$ and calculated in SPSS 25.0. Health status (pain, body awareness, coping and understanding bio-psychosocial influences on health) was compared pre- and post-treatment. Pearsons chi $^{2}$ was used to test gender-based differences in relation to experience of war and torture. Paired-samples t-tests were used to compare pain severity and pain interference, BAS-E indicators and self-rated health before and after treatment.

Qualitative interviews were conducted with seven physiotherapists, receiving training in the Pain School manual, in December 2019 and January 2020. It was attempted to achieve an equal distribution of participating NGOs and male and female respondents from the different countries (Jordan, Lebanon, Tunis).

Inclusion criteria for receiving Pain School treatment and for this study were; exposure to war trauma and/or torture, being above the age of 18 years, and referral to physiotherapy treatment due to pain complaints lasting more than 3 months. Exclusion criteria were severe psychological disorders (such as personality disorders or ongoing psychosis) or severe cognitive or mental impairment. No specific protocol for this assessment was made, since this was pre-assessed (data not available). Patients receiving less than six sessions of treatment were also excluded in this study. The reasons for dropouts were analyzed and found to be due to staff turnover during the project and patients lacking funds for transportation to and from treatment. These dropouts are not explored further in this study. The 38 patients included came from Jordan and Morocco. 
Interviews thematic analyses: A semi-structured interview guide with 20 items related to "learning objectives," "working with a manual" and "evaluation of the training," was used. The interviews were carried out online, with interpreters present at three of the seven interviews.

All interviews were recorded and transcribed. Data was analyzed using thematic analysis (Skovdal \& Cornish, 2015).

\section{Results}

\section{Qualitative evaluation of treatment and pain school training program}

The themes of the qualitative analysis from the interviews of the physiotherapists are given in the sections following below.

Theme 1. The bio-psychosocial understanding of pain: The physiotherapists expressed that they learned new ways to understand pain mechanisms and how any type of pain experience is always produced by the brain. It also provided them with a broader perspective on how to conceptualize pain. One said: "The training has given me a new perspective to how environmental, social, physical and psychosocial factors can influence pain intensity" Additionally, they mentioned that they became increasingly aware of the close linkage between stress and pain. For instance, one physiotherapist had a patient who constantly feared that the pain was caused by a serious condition.

The new knowledge helped the physiotherapist to address the constant fear as a stressor, and how that increases the severity of the pain. The biopsychosocial approach to physiotherapy and rehabilitation was new to most of the physiotherapists "Usually, we just worked with the patient as a body."

The increased focus on psychological aspects means that verbal and non-verbal communication play a greater role in the meeting between physiotherapist and patient when conducting the Pain School treatment. "I have learned to better understand the language of the patient. Because the torture survivor does not always tell things, as they are, - so you have to look after other things, to better understand them." A holistic view also encompasses noticing the body language of a patient. "It is difficult for them (i.e. the patients) to tell you everything directly, so I have to interpret. Now I am looking at their behavior, body language and breathing."

Theme 2. Group versus individual treatment: Most of the physiotherapists experienced the Pain School most effective, when provided in groups over individual treatment. Breaking isolation and engaging socially are some of the behavioral changes registered by the physiotherapists. Others explained that the patients opened up, shared thoughts and feelings and expressed relief in the meeting with others, who had the same symptoms and challenges. "When I do Pain School in groups, I experience a greater focus on the interaction between the patients and feel that the focus on the persistent pain and stress condition are harder to sustain."

Theme 3. Culture and context: According to the physiotherapists, patients start noticing relief from their pain after three to four sessions. This would convince the few patients, who were reluctant in the beginning, that the treatment could work. Reluctance towards the Pain School treatment was explained as cultural differences. "Culturally, here in the Middle East, you expect a massage from the physiotherapist." "The majority liked the idea, others liked regular physiotherapy more. " However, one physiotherapist explained that torture survivors did not like to be touched because of their bad experiences with authorities touching them violently and concluded that Pain School could be an alternative to more traditional methods. Encouraging behavioral changes, for a more active lifestyle, was difficult among patients who had been sedentary 
for many years. This was especially the case in Jordan where one of the physiotherapists explained that medical doctors in Jordan advised people just to go home and rest, which is contradictive to the Pain School approach.

Theme 4. Working "manualized" and data driven learning: It is well known among clinicians that working manualized can be challenging with patients that do not always feel, act or say what fits the format of a manual. Nevertheless, the interviews showed a mutual positive experience with the final version of the manual. "In the beginning of the training I felt confused and it was difficult to know what to do in the different sessions, but now I find that the manual supports me, so I feel I know what I am doing and when."

Physiotherapists are used to working with functional tests and questionnaires. However, working with a structured standardized tool for monitoring and evaluation was new to most of them. Few found the monitoring and evaluation time-consuming, but most were motivated by it, as the improvements in patients' symptoms suddenly was measurable and detectable.

Theme 5. Participatory learning: From the combination of lectures, groupwork, roleplay, participants presentations, physical exercises and session practice, all the interviewed physiotherapists highlighted roleplay and practicing the sessions and the physical exercises, as the most supporting activities for their learning process. "Practicing the sessions were my top priority... at the end I felt relaxed and confident with my group of patients because I had done it with the other physios (i.e. physiotherapists)." It was also expressed that there was enough time to discuss, ask questions and share clinical experiences. Challenges in the learning process evolved from the interviews as well. "At first I had an issue with the PTSD in the manual. The word itself, I understood, but it is not acceptable for these patients. I experienced that some got flashback the more I explained it. We talked about it in the training and so we changed the name." Based on similar experiences among other physiotherapists, and because PTSD is not part of the Pain School treatment, it was decided to replace PTSD with "stress" related to present stressors and past traumatic experiences.

Theme 6. Supervision and evaluation of the participants: All physiotherapists were positive about the online supervision setup between the training sessions. It was stated that the supervision was a big help and it gave a sense of self-assessment to express clinical challenges. The practical and theoretical tests were well received by the physiotherapists. Most stated that it underlined the seriousness of the training, and that it forced them to study the manual in depth. Some expressed that the practical exam was more important and felt more confident in this compared to the theoretical test. A challenge expressed by some was poor internet connection and limited time for the supervision sessions, because of other project responsibilities.

Theme 7. Personal growth: All physiotherapists expressed that the body awareness exercise practice gave them tools for self-care and awareness of their own stability, sense of grounding and ability to detect and act on their own stress reactions. "On a personal level it changed me because I became more aware. It changed my posture, my body awareness and self-awareness." A conscious awareness of being a role model was also something that was mentioned as a personal growth "We cannot convince others to believe or do something, if we don't believe or do ourselves... I cannot talk about bad or good posture if I myself have a sedentary inactive lifestyle and bad posture." The opportunity to build new competencies and self-confidence was expressed by many as a result of the train- 
ing as well as feeling a sense of added value to their career paths.

Theme 8. Clinical experiences and perceived treatment effects: When the physiotherapists were asked about the differences between regular physiotherapy and the Pain School treatment, a central theme arising was that the Pain School treatment resulted in independence from the physiotherapist. "It is a more long-lasting strategy. Patients I have seen for two or three years, suddenly don't need physiotherapy anymore." Through patient education, "They know where their pain comes from, how stress affects the pain and they know how to deal with it." Another physiotherapist expressed that, "Until now, no one came back after the last session. This is very good, because I have seen these patients for one year, where they kept coming back for more."

Compared to previous approaches to treating persistent pain conditions, the difference was seen as a combination of achieved knowledge on the interaction between stress and pain, in particular caused by effective exercises and tools they can use at home. It was emphasized that the provided tools improved the patients sleep and helped them to manage everyday activities. "At the pre-assessment the patient wouldn't even initiate to fix things around his house, but at the post-assessment the patient said that he not only tried to fix things, but he actually succeeded in fixing things around the house."

The physiotherapists stressed that the body awareness exercises, and the use of metaphors in pain education had the biggest impact on patients. A complex pain theory was taken to a level where everybody could understand it through metaphors developed to the cultural context of the patients' lives. The exercises were simple, but effective, and therefore easy to implement. "The body awareness exercises are my favorite, because the patients end up being more conscious about their feelings, of their bodies, their position, how they sit, how they walk." Practicing body awareness and using pacing principles to plan daily living, supports the patients with interpreting signals from the body and thereby being able to take better care of themselves, to to be active, prevent both de-conditioning and flareups of pain and stress. "I give them exercises and tell them about pacing and graded exercises to ensure that the concept is clear to them.

Patients that did not seem to benefit from the treatment were also mentioned. One phys-

Table 3. Distribution of socio-demographic data among patients.

\begin{tabular}{lll}
\hline & $(\mathbf{n}=\mathbf{3 8})$ & $\mathbf{\%}$ \\
\hline Gender & & \\
\hline Male & 10 & $(26.3)$ \\
Female & 28 & $(73.7)$ \\
\hline Age categories & & \\
\hline $16-25$ & 1 & $(2.6)$ \\
$26-35$ & 6 & $(15.8)$ \\
$36-45$ & 14 & $(36.8)$ \\
$46-55$ & 7 & $(18.4)$ \\
$56-65$ & 3 & $(7.9)$ \\
$66-75$ & 6 & $(15.8)$ \\
Missing & 1 & $(2.6)$ \\
\hline
\end{tabular}

\begin{tabular}{lll}
\hline Nationality & & \\
\hline Iraqi & 4 & $(10.5)$ \\
Syrian & 16 & $(42.1)$ \\
Sudanese & 3 & $(7.9)$ \\
Moroccan & 11 & $(28.9)$ \\
Other & 1 & $(2.6)$ \\
Missing & 3 & $(7.9)$ \\
\hline
\end{tabular}

\begin{tabular}{lll}
\hline Marital status & & \\
\hline Single & 3 & $(7.9)$ \\
Engaged & 1 & $(2.6)$ \\
Married & 28 & $(73.7)$ \\
Divorced & 2 & $(5.3)$ \\
Widow(er) & 4 & $(10.5)$
\end{tabular}


iotherapist working in a refugee camp shared, "Not that she (patient) did not want to act (be active in the treatment sessions and at home), but she had other problems, she does not have money or a proper home.... She got better, but not like the others."

\section{Quantitative Evaluation}

Most patients included in this study were refugees who received physiotherapy Pain School treatment in Jordan $(71.1 \%)$, whereas $28.9 \%$ were local citizens receiving treatment in Morocco (Table 3). On average, patients in this study received 9.92 treatment sessions (SD: 1.7 , range $6-15$ ) with a duration of 7.97 weeks (SD: 5.4 range 1-19). 15 patients $(39.5 \%)$ were treated in refugee camps, while $23(60.5 \%)$ received Pain School in an out-patient clinic. $28(73.7 . \%)$ received individual treatment while the remaining received group-based treatment. In one case it was not indicated whether the treatment was individual, or group based. Referral to either individual or group was solely pragmatically on what was possible in each context and was not due to a clinical evaluation. No negative results from treatment were reported.

Within this sample the vast majority had experienced traumatic war events, as only one person answered "no" and one "not at all" to the question "Have you ever experienced traumatic war events." One patient did not answer the question. Nearly half (47.4\%) had been directly exposed to torture. Pearsons $X^{2-}$ test showed a statistically significant difference $(p=0.002)$ between genders in relation to torture, as approximately one third of the women $(32.1 \%)$, while all men, except one missing, reported that they had experienced torture.

A burdened target group: The mean score for self-rated health was 3.95 indicating a poor self-rated health. Many felt pain on numerous areas of the body. Out of 45 options, patients felt pain in 11.95 areas on average. On a scale from 1-10, where 10 equals the worst pain one can imagine, the average of the perceived severity of pain was 6.51 . The pain interference level was also high with a mean score at 7.02 on a scale from 1-10. In addition to participating in the Pain School treatment, 76.3\% reported receiving other types of treatment at the pre-treatment assessment and post-treatment $47 \%$. Other treatment was mainly different types of pain relief medications. No further information to explore the reason or specifics on this was available.

Table 4 show statistical low to moderate significant changes on all parameters, with high effect sizes, ranging from $\mathrm{d}=1.04$ to $\mathrm{d}=2.38$.

Table 5 also shows statistically significant changes pre- to post-treatment for all scales on the BAS-E, except experience of breathing, with low to moderate effect sizes ranging from $d=1.1$ to $d=2.2$, except for satisfaction with looks $(\mathrm{d}=.43)$.

Patients'learning and coping outcomes: To be able to benefit from Pain School, it is crucial to understand the main elements of the treatment approach. $65.8 \%$ of the patients fully believed they could influence the intensity of their pain by planning their daily activities after the Pain School treatment compared to 18.4 $\%$ before treatment (responses "completely true"). Likewise, $55.3 \%$ fully believed that they could influence their experience of stress by planning their daily activities compared to $13.2 \%$ before treatment.

Perceptions of medicine as the only method to pain relief had changed considerably during the course of treatment, as $84.2 \%$ replied "not true at all", compared to $18.4 \%$ before Pain School.

Having resignedly negative thoughts about the prospects of one's pain condition 
improved markedly after Pain School treatment, as $81.6 \%$ replied "not true at all" to the statement that "My pain is terrible and I think it's never going to get better," post-treatment, compared to $21.1 \%$ pre-treatment

\section{Discussion}

The qualitative interviews with the physiotherapists showed predominately positive attitudes towards both Pain School, as a treatment, as well as the training program. This matches the experience of the trainers, even though the first half of the training period seemed objectively confusing for most of the physiotherapists. Some challenges can be condensed from the interviews. Finding the time for supervision and having internet connections, especially when working in the refugee camps in Jordan was hard. Addressing mental health challenges, such as PTSD, was also new and overwhelming and therefore the manual was adjusted accordingly. One physiotherapist mentioned that "The word [PTSD] itself, I understood, but it is not acceptable for these patients." Whether the physiotherapist was referring to PTSD as a label for the trauma reaction or for risking flashback reactions is not clear, but nevertheless it is very important to address in future trainings. The clinical reality in which the implementation of the Pain School treatment took place did not allow for diagnostic assessment, due to lack of economic resources

Table 4. Paired T-tests comparing Pre- and Post-treatment on BPI, Body Awareness and Self Rated Health

\begin{tabular}{lllll}
\hline & $\begin{array}{l}\text { PRE } \\
\text { Mean (SD) }\end{array}$ & $\begin{array}{l}\text { POST } \\
\text { Mean (SD) }\end{array}$ & p-value & Cohen's d \\
\hline Pain severity & $6.51(1.53)$ & $2.41(1.89)$ & $<0.001^{\star \star}$ & 2.38 \\
Pain interference & $7.02(1.79)$ & $2.64(2.31)$ & $<0.001^{\star \star}$ & 2.12 \\
Body awareness & $1.78(0.71)$ & $0.89(0.70)$ & $<0.001^{\star \star}$ & 1.26 \\
Self-rated health & $3.95(0.96)$ & $2.87(1.12)$ & $<0.001^{\star \star}$ & 1.04 \\
Number of painful & $11.95(6.67)$ & $4.76(4.00)$ & $<0.001^{\star \star}$ & 1.31 \\
body areas & & & &
\end{tabular}

Table 5. Paired T-test comparing Pre- and Post-treatment on BAS-E

\begin{tabular}{lllll}
\hline & $\begin{array}{l}\text { PRE } \\
\text { Mean (SD) }\end{array}$ & $\begin{array}{l}\text { POST } \\
\text { Mean (SD) }\end{array}$ & p-value & Cohen's d \\
\hline Muscle tension & $2.32(0.57)$ & $1.03(0.59)$ & $<0.001^{\star \star}$ & 2.22 \\
Pain or aching & $2.05(0.57)$ & $1.08(0.67)$ & $<0.001^{\star \star}$ & 1.56 \\
Day to day limitations & $1.95(0.70)$ & $1.05(0.66)$ & $<0.001^{\star \star}$ & 1.32 \\
Exercise & $1.78(0.82)$ & $0.89(0.77)$ & $<0.001^{\star \star}$ & 1.12 \\
Satisfaction with looks & $0.79(0.88)$ & $0.45(0.69)$ & $=0.003^{\star}$ & 0.43 \\
Experience of breathing & $1.18(1.04)$ & $0.89(0.65)$ & $=0.086$ & -
\end{tabular}


and clinical know-how. Nevertheless, indicators of mental health conditions should be added to the M\&E tool.

Not having a final manual to follow from the beginning and having to learn and integrate both exercise therapy and education into their treatments seemed, for many, overwhelming. The discussions and feedback during this period contributed to a manual that, to the greatest extent possible, reflected the needs of the context in the given program.

The physiotherapists found the online supervisions supportive, which also gave the trainer a chance to evaluate the clinical reasoning and implementation challenges. Even so, an offer on in-person supervision would have given a clearer idea of how the Pain School manual was used and how replicable the treatment is. Pragmatically at this phase, this was not possible, but this should be considered in the next phase.

The qualitative analysis also indicated that the training on a biopsychosocial understanding of pain, was not part of the standard educational physiotherapy programs in the Middle East that the physiotherapists had undertaken. This, and basic knowledge on trauma-related mental health conditions, should continuously be addressed when providing future trainings for physiotherapists in this region. Also, physiotherapy literature in Arabic was described as almost non-existant. The students are, in most cases, referred to medical or English and French written literature. A language barrier was described. Recently published teaching materials "Beyond Pain," an evidence-based pain science and assessment in Kurdish and Arabic (Gamble, 2020), and a 2014 praxis paper "Interventions for physiotherapist working with torture survivors" (Nielsen, 2014) in Arabic and French, are relevant contributions.

This study showed no clear indications on whether the Pain School treatment provided for individuals or groups had different effects. This has to be further investigated in the next phase of the research.

It was neither a priority, nor a possibility in this study, to isolate the patients from other types of treatments. This could be solved in the future with a control group given treatment "as usual." It would also be beneficial if the Pain School treatment were to be tested in combination with a psychotherapeutically PTSD-focused intervention, such as prolonged exposure, narrative exposure therapy, or EMDR, as long as such intervention would be ACT-compatible. The BAS-E measurement was also not validated for this population and this should be done in the future.

\section{Limitations}

A limitation in this study is that the interviews were planned, carried out and analyzed by two of the authors. One was also the trainer and the developer of the Pain School manual and a second having developed the M\&E tool. This could have influenced the physiotherapists towards a more positive attitude to both treatment and training and the authors ability to recognize answers from each respondent. Further, interviews with patients would have strengthened the study outcomes and must be included in the next phase of study.

The effect of treatment should be reviewed with caution since no control group was used .

The Pain School protocol, the selected evaluation-scales and particularly the MENA sample, makes it very hard to compare outcomes to previous studies since very few similar studies have been carried out. Also, depression, anxiety or PTSD were not measured in this study, making it unclear whether the patients were, in fact, trauma-affected from their experience of war and-or torture. 


\section{Conclusion}

This study showed good feasibility and high acceptance among the participating physiotherapists. Monitoring and evaluation of treatment was found useful, but indications of mental health status were evaluated to be missing. The pre- to post- treatment evaluation of Pain School also gave an indication of positive treatment effects. Due to the limitations, the results do not provide a final conclusion on the training program nor treatment effects. Nevertheless, this study is a first step to offer evidence-based standardized treatment for pain and trauma-affected populations in the MENAregion.

\section{Phase III and IV: Evaluation and Implementation}

Based on the results of this paper, a future phase III is planned, in addition to collecting data in a larger sample, to include: 1) Identification of socio-demographics that might show predictors in treatment outcome and if the treatment response differs when it is delivered individually or in a group 2) Add mental health-related outcomes indicating measures on trauma and persistent pain, such as PTSD, anxiety and depression. 3) Evaluate patients' subjective treatment effects and tolerability through interviews. 4) Further review and enhance the treatment manual. 5) Compare Pain School treatment with a control group receiving treatment as usual. 6) Conduct a baseline study assessing the prevalence of coexisting persistent pain and post-traumatic stress conditions in refugee populations in the MENA region.

Phase III should contain trainings of an additional two groups of physiotherapists in the region, with participants from Tunisia, Jordan, Kurdistan Iraq and Iraq. Data collection on treatment effects will be gathered in 2021 with an expected larger sample size. The 2021 train- ings will, expectedly, be conducted in Arabic and Kurdish Sorani, by local physiotherapists.

Phase IV is planned to contain final evaluation and implementation in the MENA region. An additional ambition in the implementation phase is also to explore collaborations with physiotherapy faculties in the MENA region. This will secure sustainability for future trainings in the region and further contextualization.

Acknowledgements: To all the participating physiotherapists for their contribution throughout the development of the Pain School manual. Acknowledgements also to the patients that received an unknown type of treatment. Finally, to Director Dr. Ibrahim Arqel and Head of Field Operations, Esam Albarahmeh of Noor al Hussein Foundation, Institute of Family Health, IFH Jordan, for their continuous support.

\section{References}

Abbey, H. (2015). Multidisciplinary biopsychosocial rehabilitation for chronic low back pain:

Cochrane systematic review and meta-analysis. International fournal of Osteopathic Medicine, 18(3), 239-240. https://doi.org/10.1136/bmj. h444.

Amris, K., \& Jansen, G. B. (2019). Chronic pain as a consequence of torture: management. IASP.

Factsheet no. 13. https://europeanpainfederation. eu/wp-content/uploads/2019/02/13-Chronicpain-as-consequence-of-torture-managementTEMPLATE.pdf

Asmundson, G., \& Katz, J. (2009). Understanding the co-occurrence of Anxiety Disorders and Chronic Pain: State-Of-The-Art - A Theoretical Review. Wiley-Liss, inc. https://doi.org/10.1002/ da. 20600

A-Tjak, J. G., Davis, M. L., Morina, N., Powers, M. B., Smith, J. A., \& Emmelkamp, P. M. (2015). A metanalysis of the efficacy of acceptance and commitment therapy for clinically relevant mental and physical health problems. Psychotherapy and Psychosomatics, 84(1), 30-6. https://doi.org/10.1159/000365764

Baird, E., Williams, A. C. D. C., Hearn, L., \& Amris, K. (2017). Interventions for treating persistent 
pain in survivors of torture. Cochrane Database of systematic Reviews, (8), 1-32. https://doi. org/10.1002/14651858.CD012051.pub2

Blaauwendraat, C., Berg, A. L., \& Gyllensten, A. L. (2017). One-year follow-up of basic body awareness therapy in patients with posttraumatic stress disorder. A small intervention study of effects on movement quality, PTSD symptoms, and movement experiences. Physiotherapy Theory and Practice, 33(7). https://doi.org/10.1080/0959 3985.2017.1325957

Boakye, P.A., Olechowski, C., Rashiq, S., Verrier, M. J., Kerr, B., Witmans, M., Baker, G., Joyce, A., \& Dick, B. (2016). A critical review of neurobiological factors involved in the interactions between chronic pain, depression and sleep disruption. The Clinical fournal of Pain, 32(4), 327-336. https://doi.org/10.1097/ AJP.0000000000000260

Bravo, C., Skjaerven, L. H., Espart, A., SeinEchaluce, L. G., \& Calalan-Matamoros, D. (2018). Basic Body Awareness Therapy in patients suffering from fibromyalgia: A randomized clinical trial. Physiotherapy Theory and Practice, 35(10). https://doi.org/10.1080/095 93985.2018.1467520

Brennstuhl, M. J., Tarquinio, C., \& Motel, S. (2014). Chronic pain and PTSD: Evolving views on their comorbidity. Perspectives in Psychiatric Care, 51(4), 295-304. https://doi.org/10.1111/ppc.12093

Buhman, C., Mortensen, E. L., Lundstrøm, S., Ryberg, J., Nordentoft, M., Ekstrøm, M. (2014). Symptoms, quality of life and level of functioning of traumatized refugees at Psychiatric Trauma Clinic in Copenhagen. Torture, 24(1). https:// doi.org/10.7146/torture.v24i1.109717

Chipchase, L., Sheffied, D., \& Hill, P. (2012). The long-term effectiveness of pain management programs: A systematic review and meta-analysis. Fournal of Pain Management 5(3), 215-230.

Cleeland, C. S. (1991). Brief Pain Inventory, BPI Short Version. MDAnderson Cancer Center. https:// www.mdanderson.org/research/departmentslabs-institutes/departments-divisions/symptomresearch/symptom-assessment-tools/brief-paininventory.html

Cleeland, C. S., \& Ryan, K. M. (1994). Pain assessment: global use of the Brief Pain Inventory. Ann Acad Med Singapore, 23(2), 129138.

Craig, P., Dieppe, P., Macintyre,S., Michie,S., Nazareth, I., \& Petticrew, M. (2008). Developing and evaluating complex interventions: the new Medical Research Council guidance. BMF, 29, 337. https://doi.org/10.1136/bmj.a1655
Dahl, J., \& Lundgren, T. (2006). Living Beyond Your Pain - Using Acceptance and Commitment Therapy to ease chronic pain. New Harbinger Publications.

Dahl J., Wilson, K. G., \& Nilsson, A. (2004). Acceptance and Commitment Therapy and the treatment of persons at risk for longterm disability resulting from stress and pain symptoms: A preliminary randomized trial. Behavior Therapy, 35, 785-802. https://doi. org/10.1016/S0005-7894(04)80020-0

Defrin, R., Lahav, Y., \& Solomon, Z. (2017). Dysfunctional Pain Modulation in Torture Survivors: The mediating effect of PTSD. The Fournal of Pain: Official Fournal of the American Pain Society 18(1), 1-10. https://doi. org/10.1016/j.jpain.2016.09.005

Gamble, A. (2020) Beyond Pain - An evidence-based and multilingual biopsychosocial pain science training and treatment manual. https://www.dignity.dk/en/ beyond-pain/

Gyllensten, A. L., \& Mattsson, M. (2011). Body awareness scale, movement quality and experience, BAS MQ-E. (BAS Rörelseskvalitet och Kroppsupplevelse). Simrishamn.

Gyllensten, A. L., Skoglund, K., \& Wulf., I. (2018). Basic body awareness therapy: embodied identity. Vulcan.

Harlacher, U., Nordin, L., \& Polatin, P. (2016). Torture survivors' symptom load compared to chronic pain and psychiatric in-patients. Torture, 26, (2). https://doi.org/10.7146/torture. v26i2.108204

Hedlund, L., Gyllensten, A. L., Waldegren T., \& Hansson, L. (2016). Assessing movement quality in persons with severe mental illness - reliability and validity of the body awareness scale movement quality and experience. Physiotherapy Theory and Practice. https://doi.org/10.3109/095 93985.2015.1138008

Husak, A. J., \& Bair, M. J.(2020). Chronic pain and sleep disturbances: A pragmatic review of their relationships, comorbidities, and treatments. Pain Medicine, 21(6), 1142-1152. https://doi. org $/ 10.1093 / \mathrm{pm} / \mathrm{pnz} 343$

Idler, E. L., \& Benyamini, Y. (1997). Self-rated health and mortality: A review of twentyseven community studies. Fournal of Health and Social Behavior, 38(1), 21-37. https://doi. org/10.2307/2955359

IASP, International Association of the Study of Pain, (2019). IASP Terminology. https:// www.iasp-pain.org/Education/Content. aspx?ItemNumber $=1698$ \#Pain

Kim H. J., \& Yu, S. H. (2015). Effects of complex manual therapy on PTSD, pain, function, and 
balance of male torture survivors with chronic low back pain. Fournal of Physical Therapy Science 27(9), 2763-66. https://doi.org/ 10.1589/ jpts. 27.2763

Liedl, A., Müller, J., Morina, N., Karl, A., Denke, C., \& Knaevelsrud C. (2011). Physical activity within a CBT intervention improves coping with pain in traumatized refugees: results of a randomized controlled design. Pain Medicin, 12, 234-45. https://doi.org/10.1111/j.1526-4637.2010.01040

Louw, A. , Zimney, K., Puentedura., E. J., \& Diener, I. (2016). The efficacy of pain neuroscience education on musculoskeletal pain: A systematic review of the literature. Physiother Theory Pract. 5, 332-55. https://doi.org/10.1080/09593985.20 16.1194646

Malfliet, A., Kregel, J., Meeus, M., Roussel, N., Danneels, L., Cagnie, B., Mieke, D., \& Nijs, J. (2018). Blended-learning pain neuroscience education for people with chronic spinal pain: Randomized controlled multicenter trial. Physical Therapy, 98 (5), 357-368. https://doi. org/10.1093/ptj/pzx092

Martinez-Calderon, J., Flores-Cortes, M., MoralesAsencio, J. M., \& Luque-Suarez, A. (2019). Pain-related fear, pain intensity and function in individuals with chronic musculoskeletal pain: A systematic review and meta-analysis. The Fournal of Pain, 20, (12),1394-1415. https://doi. org/10.1016/j.jpain.2019.04.009

McAndrew, L. M., Lu, S. E., Phillips, L. A., Naestro, K., \& Quigley, K. S. (2019). Mutual maintenance of PTSD and physical symptoms for veterans returning from deployment. European fournal of Psychotraumatology, 10. https://doi.org/ 10.1080/20008198.2019.1608717

McGrath, M., Acarturk, C., Roberts, B., Ilkkursun, Z., Sondorp, E., Sijbrandij, M. P., Cuijpers, P., Ventevogel, P., McKee, M., \& Fuhr, D. C. (2020). Somatic distress among Syrian refugees in Istanbul, Turkey: A cross-sectional study. Fournal of Psychosomatic Research, 132. https:// doi.org/10.1016/j.jpsychores.2020.109993

Moseley, G. L., \& Butler, D. S. (2013). Explain Pain. Second edition. Noigroup publications.

Moseley, G. L., \& Butler, D. S. (2015). Fifteen Years of Explaining Pain: The past, present and the future. The fournal of Pain, 16(9), 807-813. https://doi.org/10.1016/j.jpain.2015.05.005

Moseley G. L. \& Butler D. S. (2017). Explain Pain Supercharged. Noigroup publications.

Nielsen, H. F. (2014). Interventions for physiotherapist working with torture survivors - A DIGNITY praxis paper. DIGNITYDanish Institute against Torture, 6 \& 31 . https:// www.dignityinstitute.org/resources/otherpublications/international- publication-series/ dignity-publicationn-series-on-torture-andorganised-violence-no-6/

Nicol, A. L., Sieberg, C. B., Clauw, D. J., Hassett, A. L., Moser, S. E., \& Brummett C. M. (2016). The association between a history of lifetime traumatic events and pain severity, physical function, and affective distress in patients with chronic pain. The fournal of Pain, 17, (12), 1334-1348. https://doi.org/10.1016/j. jpain.2016.09.003

Nordin, L \& Perrin, S. (2019). Pain and posttraumatic stress disorder in refugees who survived torture: The role of pain catastrophizing and trauma-related beliefs. Eur F Pain, 23, 14971506. https://doi.org/10.1002/ejp.1415

Nordin, L. (2020) Posttraumatic stress reactions in tortured refugees. Relationship to pain, cognitive impairment, and negative appraisals. Faculty of Social Sciences, Department of Psychology, Lund University. https://portal.research.lu.se/portal/ en/publications/posttraumatic-stress-reactionsin-tortured-refugees(98871365-c64a-4fd4-8fedcbc3df1be8c3).html

Nyboe, L., Bentholm A., \& Gyllensten, A. L. (2016). Bodily symptoms in patients with posttraumatic stress disorder: A comparative study of traumatized refugees, Danish war veteran, and healthy controls. Fournal for Bodywork $\mathcal{E}$ Movement Therapies, 21(3), 523-527. https://doi. org/10.1016/j.jbmt.2016.08.003

Pacella, M., Hruska, B., \& Delahanty, D. L. (2013). The physical health consequences of PTSD and PTSD symptoms: A meta-analytic review (2013). Fournal of Anxiety Disorders, 27, 33-46. https:// doi.org/ 10.1016/j.janxdis.2012.08.004

Pardo, G. B., Girbés, E. L., Roussel, N. A., Izquierdo, T. G., Penick, V. J., \& Martín, D. P. (2018). Pain neurophysiological education and therapeutic exercise for patients with chronic low back pain: A Single-blind randomized controlled trial. Physical Medicine and Rehabilitation, 99, 338-347. https://doi.org/10.1016/j.apmr.2017.10.016

Perrot, S., Cohen, M., Barke, A., Korwisi, B., Rief, W., \& Treede, R. (2019). The IASP Taskforce for the Classification of Chronic Pain. PAIN, 160(1),77-82. https://doi.org/ 10.1097/j. pain.0000000000001389

Probst, M., Skjaerven, L. H. (2018). Physiotherapy in Mental Health and Psychiatry - a scientific andclinical based approach. Elsevier Ltd.

Rometsch-Ogioun El Sount, C., Windthorst, P., Denkinger, J., Ziser, K., Nikendei, C., Kindermann, D., Ringwald, J., Renner, V., Zipfel, 
S., \& Junne. (2019). Chronic pain in refugees with posttraumatic stress disorder (PTSD): A systematic review on patients ' characteristics and specific interventions. $\mathcal{F}$ Psychosom Res, 118, 83-97. https://doi.org/10.1016/j. jpsychores.2018.07.014

Schnittker, J., \& Bacak, V. (2014). The increasing predictive validity of self-rated health. PloS One, 9(1). https://doi.org/10.1371/journal. pone. 0084933

Skovdal, M., Cornish, F. (2015) Qualitative research for development: a guide for practitioners. Practical Action Publishing.

Stade, K., Skammeritz, S., Hjortkjær, C., \& Carlsson, J. (2015). "After all the traumas my body has been through, I feel good that it is still working." - Basic Body Awareness Therapy for traumatized refugees. Torture, 25(1). https://doi.org/10.7146/ toture.v25i1.109507

Sundén, A., Ekdahl, C., Horstman, V., \& Gyllensten, A. L. (2016). Analyzing movement development and evaluation of Body Awareness Scale Movement Quality (BAS MQ- E). Physiother. Res. Int. 21(2), 70-76. https://doi. org/10.1002/pri.1618

Tan, G., Jensen, M. P., Thornby, J. I., \& Shanti, B. F. (2004). Validation of the Brief Pain Inventory for chronic nonmalignant pain. $\mathcal{F}$ Pain, 5(2), 133137. https://doi.org/10.1016/j.jpain.2003.12.005

Tsang, A., Korff, M. V., Lee, S., Alonso, J., Karam, E., Angermeyer, M. C., Borges, G. L. G., Bromet, E. J., Girolamo, O., Graaf, R., Gureje, O., Lepine, J., Haro, J. M., Levinson, D., Oakley Browne, M. A., Posada-Villa, J., Seedat, S., \& Watanabe, M. (2008). Common Chronic Pain Conditions in Developed and Developing Countries: Gender and Age Differences and Comorbidity with Depression-Anxiety Disorders. The fournal of Pain, 9, (10), 883-891. https://doi. org/10.1016/j.jpain.2008.05.005

Turk, D. C., Dworkin, R., H., Allen, R. R., Bellamy, N., Brandenburg, N., Carr, D. B., \& Witter, J. (2003). Core outcome domains for chronic pain clinical trials: IMMPACT recommandations. Pain, 106(3), 337-345. https://doi.org/10.1016/j. pain.2003.08.001

Vlaeyen, J. W., \& Linton, S. J. (2000). Fear-avoidance and its consequences in chronic musculoskeletal pain: a state of the art. Pain, 85(3), 317-332. https://doi.org/10.1016/s0304-3959(99)00242-0

Wang, S. J. (2012). Effectiveness of combined intervention approaches in the treatment of affective disorders and chronic pain in war victims. ClinicalTrials. NCT01696578. https:// clinicaltrials.gov/ct"/show/NCTO1696578
Williams, A. C., \& Alayarian, A. S. (2019) Chronic pain as a consequence of Torture: Assessment. 2019. Global year against pain in the most vulnerable. IASP. http:// s3.amazonaws.com/rdcms-iasp/files/ production/public/2019GlobalYear/Fact Sheets $/ 12 \% 20$ Chronic\%20Pain $\% 20$ as $\% 20$ a $\% 20$ Consequence $\% 20$ of $\% 20$ Torture $\% 20$ Assessment $\% 20$ RUS.pdf

World Medical Association (WMA). (2013). Declaration of Helsinki: Ethical Principles for Medical Research Involving Human Subjects. Ferney-Voltaire, France. (2013). https://www. wma.net/policies-post/wma-declaration-ofhelsinki-ethical-principles-for-medical-researchinvolving-human-subjects/

Zhenggang, B., Shiga, L., Luyao, Z., Sijie, W., \& Iris, C. (2020). Acceptance and Commitment Therapy (ACT) to reduce depression: A systematic review and meta-analysis. Fournal of Affective Disorders, 260(1), 728-737. https://doi. $\operatorname{org} / 10.1016 /$ j.jad.2019.90.040 